\title{
Predictive models in software engineering
}

\author{
Tim Menzies • Gunes Koru
}

Published online: 12 April 2013

(C) Springer Science+Business Media New York 2013

Welcome to the Empirical Software Engineering's special issue on predictive models in software engineering. The goal of such methods is repeatable, refutable (and possibly improvable) results in software engineering.

Many of the recent papers in SE literature are based on data from on-line repositories such as http://promisedata.googlecode.com. This introduces a kind of selection in the kinds of papers published at this venue. Our first paper pushes past that bias to explore a very rich time-based data set. In "Predicting the Flow of Defect Correction Effort using a Bayesian Network Model", Schulz et al. use a Bayes net to explore the effects of removing defects at different stages of the software lifecycle. Their work shows how to calibrate general models to the particulars of a company's local particulars.

Our next paper "The Limited Impact of Individual Developer Data on Software Defect Prediction" by Bell et concludes there is no added value to reasoning on some aspects of social aspects of programmer teams working on a code. This is a timely counterpoint to other research that eschews code measures for other approaches based only on social metrics.

Our last paper explores the complicated issue of parameter tuning. In "Using Tabu Search to Configure Support Vector Regression for Effort Estimation”, Corazza et al. offers automated guidance for setting the parameters that control a learner. This is a matter of critical importance since even the best learner can perform poorly if its operator uses the wrong settings.

A special issue like this is only possible due to the hard work of a dedicated set of authors are reviewers. We would like to express our gratitude to all authors who submitted their papers this special issue. We would also like to thank our reviewers for their meticulous evaluation of the submissions. The success of special issues such as this one largely stands on their shoulders.

T. Menzies $(\bowtie)$

West Virginia University, Morgantown, USA

e-mail: tim@menzies.us

G. Koru

University of Maryland, Baltimore County, Baltimore, USA

e-mail: gk@drkoru.us 


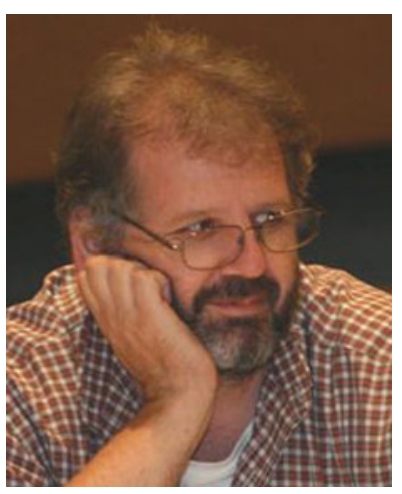

Tim Menzies is a Professor in CS at WVU and the author of over 200 referred publications. In terms of citations, he is one of the top 100 most most cited authors in software engineering (out of 54,000+ researchers, see http://goo.gl/vggy1). At WVU, he has been a lead researcher on projects for NSF, NIJ, DoD, NASA, as well as joint research work with private companies. He teaches data mining and artificial intelligence and programming languages. Prof. Menzies is the co-founder of the PROMISE conference series (along with Jelber Sayyad) devoted to reproducible experiments in software engineering: see http:// promisedata.googlecode.com. He is an associate editor of IEEE Transactions on Software Engineering, the Empirical Software Engineering Journal, and the Automated Software Engineering Journal. For more information, see http://menzies.us.

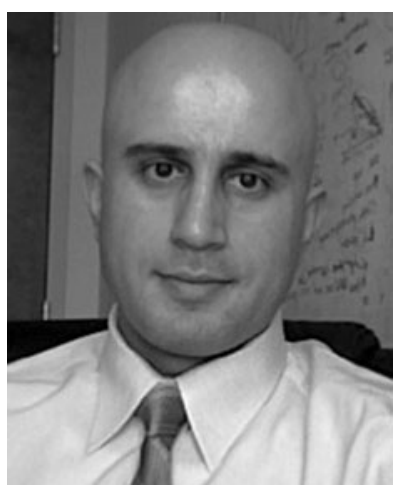

Gunes Koru is an associate professor in the Department of Information Systems at the University of Maryland, Baltimore County (UMBC). He joined the PROMISE community in 2005, and he has contributed many data sets to the PROMISE data repository since then. In 2010, he served as the program chair of the PROMISE 2010 conference.

His research interests fall into the areas of software engineering and health information systems. 